\title{
Sleep experiences during different lifetime periods and in vivo Alzheimer pathologies
}

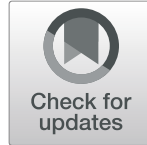

Young Min Choe ${ }^{1}$, Min Soo Byun², Dahyun Yi ${ }^{2}$, Jun Ho Lee ${ }^{3}$, So Yeon Jeon ${ }^{4}$, Bo Kyung Sohn ${ }^{5}$, Yu Kyeong Kim6, Seong A Shin ${ }^{6}$, Chul-Ho Sohn ${ }^{7}$, Yu Jin Lee ${ }^{8,9}$, Dong Young Lee ${ }^{3,9^{*}}$ (D) and for the KBASE Research Group

\begin{abstract}
Background: Very little is known for the direction or causality of the relationship between lifetime sleep experiences and in vivo Alzheimer's disease (AD) pathologies. This study aimed to examine the relationship between sleep experiences during the young adulthood, midlife, and late-life periods and in vivo cerebral beta-amyloid (A $\beta$ ) deposition and AD signature regional neurodegeneration in cognitively normal (CN) old adults.

Methods: This study included 202 CN old adults who participated in the Korean Brain Aging Study for the Early Diagnosis and Prediction of Alzheimer's Disease (KBASE) study. All participants underwent a comprehensive clinical assessment, $\left[{ }^{11} \mathrm{C}\right]$ Pittsburgh Compound B positron emission tomography (PET), $\left[{ }^{18} \mathrm{~F}\right]$ Fluorodeoxyglucose-PET, and magnetic resonance imaging. The quality and duration of sleep were assessed for the following age periods: 20 30s, 40-50s, and the most recent month. All analyses were adjusted for age, gender, education, apolipoprotein E $\varepsilon 4$ status, vascular risk score, Hamilton Depression Rating Scale score, and use of sleep medication.
\end{abstract}

Results: Bad sleep quality and short sleep duration during midlife were significantly associated with increased $A \beta$ deposition and $A D$ signature regional hypometabolism, respectively. Although current bad sleep quality appeared to be associated with increased $A \beta$ accumulation, this association disappeared after controlling for the effects of midlife sleep quality. Neither the quality nor duration of sleep during young adulthood was related to $A \beta$ burden or neurodegeneration.

Conclusions: Bad sleep quality during midlife increases pathological $A \beta$ deposition in the brain, while short sleep duration during the same period accelerates regional hypometabolism.

Keywords: Midlife sleep, Preclinical Alzheimer's disease, Cerebral amyloid, Neurodegeneration

\section{Background}

A number of studies have shown that disturbed sleep and Alzheimer's disease (AD) dementia are closely related [1-3]. Further, experimental evidence suggests that the relationship between poor sleep and beta-amyloid $(A \beta)$ accumulation, which is a pathological hallmark of $\mathrm{AD}$, is bidirectional [4]. For example, $\mathrm{A} \beta$ aggregation disrupts the sleep-wake cycle as well as the diurnal fluctuation of $A \beta$ in mice [5], and conversely, acute sleep deprivation increases brain $A \beta$ levels and chronic sleep restriction increased $A \beta$ plaque formation in transgenic

\footnotetext{
* Correspondence: selfpsy@snu.ac.kr

${ }^{3}$ Department of Neuropsychiatry, Seoul National University Hospital, Seoul, Republic of Korea

${ }^{9}$ Department of Psychiatry, Seoul National University College of Medicine, Seoul, Republic of Korea

Full list of author information is available at the end of the article
}

mice [6]. It has also been suggested that AD pathophysiology impairs the sleep-regulating system including hypocretin and melatonin regulation, and reversely, hypocretin and melatonin may play an important role in modulating $\mathrm{AD}$ pathophysiology [7].

Several clinical studies based on amyloid positron emission tomography (PET) imaging have revealed that poorer self-reported sleep quality is associated with a greater in vivo $A \beta$ burden in the human brain [8-11], while the association between self-reported sleep duration and $A \beta$ deposition is controversial $[8,10-12]$. However, all studies that have investigated this relationship had cross-sectional designs and merely correlated current sleep experience with $A \beta$; thus, they could not provide any clue on the direction or causality of the relationship. Two translational human studies partly demonstrated the causal relationship; 
one night of sleep deprivation increased cerebrospinal fluid (CSF) A 342 [13], and slow-wave activity disruption increased CSF A $\beta 40$ [14]. However, they only tested the acute effect of disrupted sleep, which may differ from the lifelong effects of poor sleep.

To overcome these limitations, we assessed sleep experiences, including sleep quality and sleep duration, of cognitively normal $(\mathrm{CN})$ old adults during three periods of life: young adulthood, midlife, and late-life. Rather than merely assessing the cross-sectional association between current sleep and pathology, this information was used to examine the influences of each lifetime sleep experience on in vivo cerebral $\mathrm{A} \beta$ deposition. In addition, the associations of lifetime sleep experiences with $\mathrm{AD}$ signature regional neurodegeneration, as measured using cerebral glucose metabolism and cortical thickness, were evaluated. Only limited information is available regarding this association $[9,15]$.

\section{Methods \\ Participants}

The present study was part of the Korean Brain Aging Study for the Early Diagnosis and Prediction of Alzheimer's Disease (KBASE) [16], which is an ongoing prospective cohort investigation, and included a total of $202 \mathrm{CN}$ elderly individuals from the KBASE cohort. The inclusion criteria were being aged from 60 to 90 years old, a Clinical Dementia Rating score of 0 , and no diagnosis of mild cognitive impairment or dementia. The exclusion criteria were as follows: the presence of any serious medical, psychiatric, or neurological disorder that could affect mental functioning; major sleep disorder other than insomnia disorder (e.g., rapid eye movement sleep behavior disorder, obstructive sleep apnea, and/or restless legs syndrome); the presence of severe communication problem that would make a clinical examination or brain scan difficult; contraindications for a magnetic resonance imaging (MRI) scan; the absence of a reliable informant; illiteracy; and/or participation in another clinical trial of sleep treatment.

\section{Clinical assessment}

All participants underwent a standardized clinical evaluation conducted by trained psychiatrists which was based on the KBASE clinical assessment protocol, which incorporates the Korean version of the Consortium to Establish a Registry for Alzheimer's Disease Assessment Packet (CERAD-K) [17]. The KBASE neuropsychological assessment protocol that incorporates the CERAD neuropsychological battery [18] was also given to all participants by trained neuropsychologists. The presence of six vascular risk factors, including hypertension, diabetes, dyslipidemia, transient ischemic attack, stroke, and coronary artery disease, was systematically evaluated; a vascular risk score (VRS) was calculated as the total number of the vascular risk factors and reported as a percentage [19]. Depressive symptoms were measured using the Hamilton Depression Rating Scale (HDRS) [20].

\section{Assessment of sleep experiences}

All participants were systematically interviewed by trained nurses using a structured interview form to determine past average sleep duration and subjective sleep quality during the following lifetime periods: 20-39 (young adulthood) and 40-59 (midlife) years. The participants also completed the Pittsburgh Sleep Quality Index (PSQI) [21]; of the 18 PSQI items, items assessing sleep duration (item \#4) and subjective sleep quality (item \#18) were used for the evaluation of current (i.e., late-life) sleep experiences. For all past (i.e., young adulthood and midlife) and current sleep experiences, sleep duration was reported as the mean hour of sleep and subjective sleep quality was rated on a 4-point scale ("very good," "fairly good," "fairly bad," and "very bad"). Information about current and past sleep disorders and the current use of sleep medication was also obtained through the structured interview and a review of medical records by trained nurses.

\section{Neuroimaging evaluation $A \beta$ deposition}

All participants underwent simultaneous three-dimensional (3D) $\left[{ }^{11} \mathrm{C}\right]$ Pittsburgh compound B (PiB) PET and 3D T1weighted MRI scans using a 3.0-T Biograph mMR (PETMR) scanner (Siemens; Washington DC, USA); the details of the PiB-PET and MRI acquisition and preprocessing have been described in a previous report from our research group [22]. The automated anatomical labeling algorithm [23] and a region-combining method [24] were applied to determine the region of interests (ROIs) to characterize

${ }^{11} \mathrm{C}-\mathrm{PiB}$ retention levels in the frontal, lateral parietal, posterior cingulate-precuneus, and lateral temporal regions of the brain. The standardized uptake value ratio (SUVR) for each ROI was calculated by dividing the mean value for all voxels within each ROI by the mean cerebellar uptake value [25]. Participants were classified as $A \beta$ positive if the SUVR was $>1.4$ in at least one of the four ROIs or as $A \beta$ negative if the SUVR each of the four ROIs was $\leq 1.4[24,26]$. A global $A \beta$ retention value was generated by dividing the mean value for all voxels of the global cortical ROI by the mean cerebellar uptake value in the same image $[24,25,27]$.

\section{$A D$ signature region neurodegeneration}

Participants also underwent $3 \mathrm{D}\left[{ }^{18} \mathrm{~F}\right]$ fluorodeoxyglucose (FDG) PET imaging using the same PET-MR machine described above. The details of the FDG-PET image acquisition and preprocessing have been described in a previous report from our research group [22]. Voxelweighted mean SUVRs were extracted from AD signature 
FDG ROIs known to be sensitive to the metabolic changes associated with $\mathrm{AD}[27,28]$, including the angular gyri, posterior cingulate cortex, and inferior temporal gyri. $\mathrm{AD}$ signature region cerebral glucose metabolism (AD-CM) was defined as the voxel-weighted mean SUVR of the AD signature FDG ROIs. AD signature region cortical thickness (AD-CT) on the MRI scans was defined as the mean cortical thickness values obtained from AD signature regions, including the entorhinal, inferior temporal, and middle temporal cortices and fusiform gyrus, as previously described [27]. AD signature neurodegeneration (AD-ND) positivity was defined as an $\mathrm{AD}-\mathrm{CM}<1.386$ and/or an $\mathrm{AD}-\mathrm{CT}<2.666 \mathrm{~mm}$, as described in previous studies [27-29].

\section{Apolipoprotein E genotyping}

Genomic DNA was extracted from whole blood samples, and apolipoprotein E (APOE) genotyping was performed as previously described [30]. APOE $\varepsilon 4$ (APOE4) positivity was coded if at least one $\varepsilon 4$ allele was present.

\section{Statistical analyses}

For the present analyses, self-reported sleep quality was originally recorded using a 4-point scale but then was transformed into a dichotomous variable ("good" or "bad") because only a small number of participants reported "very bad" sleep quality. Duration of sleep was treated as a continuous variable. Multiple logistic regression analyses were performed to examine the association between sleep quality (or sleep duration) during each lifetime period and the rate of $\mathrm{A} \beta$ (or AD-ND) positivity after controlling for age, gender, education, APOE4, VRS, current use of sleeping pills, and HDRS score. In addition, multiple linear regression analyses that treated global $\mathrm{A} \beta$ deposition and neurodegeneration biomarkers as dependent variables were conducted after controlling for the same covariates used in the logistic regression analyses. Global A $\beta$ deposition and AD-CT were natural log-transformed to achieve a normal distribution. In the event that a sleep variable was significantly related to an imaging variable, the moderating effects of APOE4 were further explored by also including a sleep variable $x$ APOE4 interaction term as an independent variable after controlling for the same covariates. All statistical analyses were conducted using IBM SPSS Statistics 21, and $p$ values $<0.05$ were considered to indicate statistical significance.

\section{Results}

\section{Demographics and clinical characteristics}

The characteristics of the study participants are presented in Table 1. After the sleep quality based on the 4-point scale was transformed into a dichotomous variable ("good" or "bad"), the proportions of bad sleep were $6.4 \%, 10.4 \%$,
Table 1 Demographics and clinical characteristics of study subjects $(n=202)$

\begin{tabular}{ll}
\hline Age, years & $71.07(6.74)$ \\
Female & $102(50.5 \%)$ \\
Education level, years & $11.51(4.77)$ \\
APOE4 carrier & $37(18.3 \%)$ \\
Vascular risk score, \% & $17.49(15.84)$ \\
HDRS & $0.74(1.51)$ \\
Current use of any sleep medication & $6(3.0 \%)$ \\
Bad sleep quality (bad + very bad) & \\
Young adulthood & $13(6.4 \%)$ \\
$\quad$ Midlife & $21(10.4 \%)$ \\
Current & $24(11.9 \%)$ \\
Sleep duration, h & \\
Young adulthood & $6.83(1.11)$ \\
Midlife & $6.65(1.18)$ \\
Current & $6.42(1.31)$ \\
A $\beta$ positive & $33(16.3 \%)$ \\
Global A $\beta$ deposition & $1.20(0.25)$ \\
AD-ND positive & $112(55.4 \%)$ \\
AD-CM & $1.41(0.12)$ \\
AD-CT, mm & $2.84(0.18)$ \\
\hline Data a presen &
\end{tabular}

Data are presented as mean (standard deviation) or number (\%) APOE4 apolipoprotein E $\varepsilon 4$, HDRS Hamilton Depression Rating Score, $A \beta$ betaamyloid, $A D-N D$ Alzheimer's disease signature region neurodegeneration, $A D$ $C M$ Alzheimer disease signature region cerebral glucose metabolism, $A D-C T$ Alzheimer's disease signature region cortical thickness

and $11.9 \%$ for the young adulthood, midlife, and current lifetime periods, respectively. Although the percentage of bad sleepers increased with age, the differences were not statistically significant (chi-square value $=2.496, \quad p=$ 0.287 ). The mean durations of sleep were $6.83,6.65$, and $6.42 \mathrm{~h}$ for each lifetime period; these differences were significant according to an analysis of variance (ANOVA; $F=6.048, \mathrm{df}=2, p=0.003)$.

\section{Associations between sleep experiences and $A \beta$ deposition}

Multiple logistic regression analyses revealed that midlife and current sleep qualities were significantly associated with $A \beta$ positivity whereas young adulthood sleep quality was not (Table 2). The bad sleep qualities in the midlife and current periods were associated with 4.636fold and 4.194-fold increases in the probability of $A \beta$ positivity, respectively. Additional analyses including the sleep quality $\times$ APOE4 interaction term revealed that the interaction term was not significant for either the midlife or current lifetime period. Sleep duration in any lifetime periods was not related to $A \beta$ positivity. As shown in Table 2, the multiple linear regression analyses revealed that midlife sleep quality had a significant 
Table 2 Association between lifetime sleep experience and A $\beta$ positivity (or global A $\beta$ deposition)

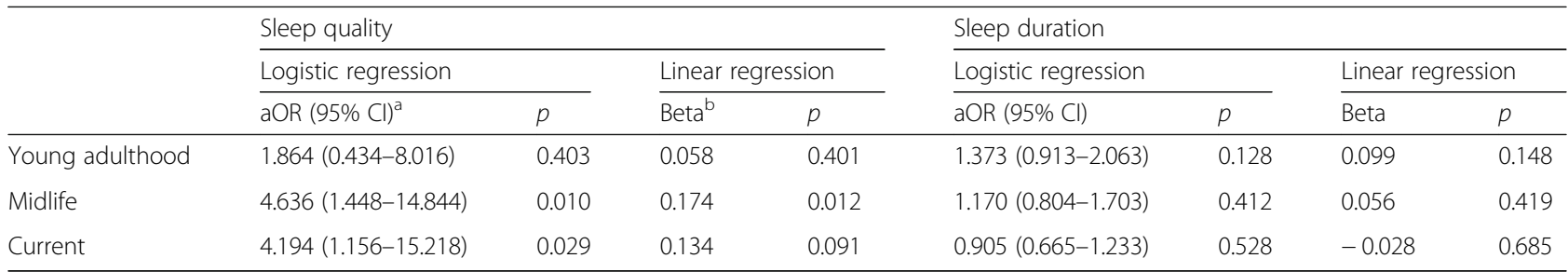

Both logistic and linear regression analyses were adjusted for age, gender, education, APOE4 status, vascular risk score, use of sleep pill, and Hamilton Depression Rating Score. Global $A \beta$ deposition was natural log-transformed to achieve normal distribution

$A \beta$ beta-amyloid, $a O R$ adjusted odds ratio, $C l$ confidence interval, $A P O E 4$ apolipoprotein $\mathrm{E} \varepsilon 4$

${ }^{a}$ Good sleep quality was taken as a reference, so aOR represents the likelihood of being $A \beta$ positive for bad sleep quality compared with good one

${ }^{b}$ Positive beta value means that worse sleep quality is associated with increased $A \beta$ deposition

association with global $\mathrm{A} \beta$ deposition. By contrast, there were no significant relationships between another lifetime sleep quality and global $\mathrm{A} \beta$ deposition or between sleep duration in any lifetime periods and $\mathrm{A} \beta$ deposition.

Table 3 shows the results from the multiple logistic regression analysis that included both midlife and current sleep qualities as independent variables and $A \beta$ positivity as a dependent variable. Midlife sleep quality was significantly associated with $A \beta$ positivity whereas late-life sleep quality was not.

\section{Associations between sleep experiences and AD signature neurodegeneration}

Multiple logistic regression analyses revealed that sleep quality in any lifetime periods was not associated with AD-ND positivity (Table 4). However, shorter sleep duration in midlife, but not the other lifetime periods, was significantly associated with a higher AD-ND positivity rate. There were no interaction effects of midlife sleep duration and APOE4 on AD-ND positivity. These results

Table 3 Multiple logistic regression analysis with midlife and current sleep qualities as independent variables and $A B$ positivity as a dependent variable

\begin{tabular}{lll}
\hline Covariates & $\mathrm{aOR}(95 \% \mathrm{Cl})$ & $p$ value \\
\hline Age & $1.112(1.041-1.188)$ & 0.002 \\
Gender & $1.043(0.424-2.563)$ & 0.927 \\
Education & $1.056(0.954-1.168)$ & 0.292 \\
APOE4 status & $3.314(1.297-8.467)$ & 0.012 \\
Vascular risk score & $0.987(0.960-1.015)$ & 0.357 \\
Use of sleep pill & $4.606(0.715-29.659)$ & 0.108 \\
HDRS & $0.833(0.572-1.212)$ & 0.340 \\
Midlife sleep quality & $3.796(1.117-12.895)^{\mathrm{a}}$ & 0.033 \\
Current sleep quality & $3.183(0.793-12.779)^{\mathrm{a}}$ & 0.103 \\
\hline
\end{tabular}

$A \beta$ beta-amyloid, $a O R$ adjusted odds ratio, $C l$ confidence interval, APOE4 apolipoprotein E $\varepsilon 4, H D R S$ Hamilton Depression Rating Scale

${ }^{\mathrm{a}} \mathrm{Good}$ sleep quality was taken as a reference, so aOR represents the likelihood of being $A \beta$ positive for bad sleep quality compared with good one were very similar, even after controlling for global $\mathrm{A} \beta$ retention as an additional covariate.

Multiple linear regression analyses revealed that bad sleep quality in any lifetime periods was not associated with either $\mathrm{AD}-\mathrm{CM}$ or $\mathrm{AD}-\mathrm{CT}$ (Table 5). In terms of sleep duration, shorter duration in midlife, but not any other lifetime periods, was significantly associated with decreased AD-CM. Additional analyses did not reveal an interaction between midlife sleep duration and APOE4 and showed that sleep duration in any lifetime periods was not related to AD-CT. Similar results were obtained after further adjustment for global $\mathrm{A} \beta$ deposition (Additional file 1: Table S1).

\section{Discussion}

In $\mathrm{CN}$ elderly individuals, bad sleep quality and shorter sleep duration during the midlife period were significantly associated with higher levels of $A \beta$ deposition and increased AD-ND, respectively. Although current bad sleep quality appeared to be associated with increased A $\beta$ accumulation, this relationship disappeared after controlling for the effects of midlife sleep quality. Neither sleep quality nor duration of sleep during young adulthood was related to $A \beta$ burden or neurodegeneration. To the best of our knowledge, this study is the first to reveal the associations of sleep experience in different lifetime periods with cerebral $\mathrm{A} \beta$ accumulation and neurodegeneration.

Based on the temporal order of the lifetime periods, the finding that there was a significant association between bad sleep quality during the 40-50 years of age period and increased late-life $A \beta$ deposition suggests that midlife sleep disturbances may contribute to pathological $A \beta$ deposition. Similarly, the significant association between short sleep duration in midlife and increased AD-ND could also be interpreted as the influence of short sleep duration during midlife on the acceleration of the neurodegenerative process. Although several previous studies have reported that a greater $A \beta$ burden is associated with poorer self-reported sleep quality in $\mathrm{CN}$ old adults [8-11], these studies only collected current sleep data at the time 
Table 4 Multiple logistic regression analyses with AD-ND positivity as a dependent variable

\begin{tabular}{|c|c|c|c|c|c|c|c|c|}
\hline & \multicolumn{4}{|l|}{ Sleep quality } & \multicolumn{4}{|l|}{ Sleep duration } \\
\hline & \multicolumn{2}{|l|}{ Model 1} & \multicolumn{2}{|l|}{ Model 2} & \multicolumn{2}{|l|}{ Model 1} & \multicolumn{2}{|l|}{ Model 2} \\
\hline & $\mathrm{aOR}(95 \% \mathrm{Cl})^{\mathrm{a}}$ & $p$ & $\mathrm{aOR}(95 \% \mathrm{Cl})^{\mathrm{a}}$ & $p$ & aOR (95\% Cl) & $p$ & aOR (95\% Cl) & $p$ \\
\hline Young adulthood & $1.428(0.426-4.786)$ & 0.564 & $1.423(0.423-4.781)$ & 0.569 & $0.826(0.628-1.088)$ & 0.174 & $0.823(0.625-1.084)$ & 0.166 \\
\hline Midlife & $1.989(0.714-5.539)$ & 0.188 & $1.999(0.708-5.641)$ & 0.191 & $0.679(0.516-0.894)$ & 0.006 & $0.678(0.514-0.893)$ & 0.006 \\
\hline Current & $0.693(0.236-2.036)$ & 0.504 & $0.677(0.227-2.018)$ & 0.484 & $0.938(0.745-1.182)$ & 0.588 & $0.939(0.745-1.184)$ & 0.59 \\
\hline
\end{tabular}

Model 1: adjusted for age, gender, education, APOE4 status, vascular risk score, use of sleep pill, Hamilton Depression Rating Score. Model 2: adjusted for covariates of model $1+$ global $A \beta$ deposition. Global $A \beta$ deposition was natural log-transformed to achieve normal distribution

$A D-N D$ Alzheimer's disease signature region neurodegeneration, $a O R$ adjusted odds ratio, $C l$ confidence interval, $A \beta$ beta-amyloid, $A P O E 4$ apolipoprotein $\mathrm{E} \varepsilon 4$

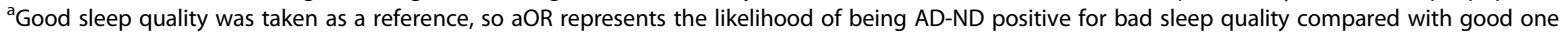

of amyloid imaging. Therefore, the results are not able to provide information regarding the direction of the relationship between sleep and $A \beta$ deposition. Similar to previous studies, we found that current sleep quality was significantly associated with $A \beta$ accumulation when not considering the effects of the past sleep experience. However, this difference was no longer significant when the effects of sleep quality over the preceding midlife period were considered in the same model. Taken together, the present results suggest that chronic bad sleep experiences, particularly poor sleep quality during midlife, could accelerate cerebral $A \beta$ deposition. However, the actual influence of current sleep quality on $A \beta$ deposition, and vice versa, remains unclear. In contrast to the results for sleep quality, there were no associations between the past or current sleep duration and $A \beta$ accumulation in the present study, which is consistent with most previous reports [10-12, 31]. This null finding can be explained, at least in part, by the fact that an optimal duration of sleep, compared to sleep quality, will vary across the lifespan and from person to person [32].

Table 5 Multiple linear regression analyses with neurodegeneration biomarkers as dependent variables

\begin{tabular}{|c|c|c|c|c|}
\hline & \multicolumn{2}{|c|}{ Sleep quality } & \multicolumn{2}{|c|}{ Sleep duration } \\
\hline & Beta $^{a}$ & $p$ & Beta $^{a}$ & $p$ \\
\hline \multicolumn{5}{|c|}{ Dependent variable: AD-CM } \\
\hline Young adulthood & 0.011 & 0.880 & 0.135 & 0.054 \\
\hline Midlife & -0.058 & 0.418 & 0.173 & 0.015 \\
\hline Current & -0.013 & 0.871 & $<0.001$ & 0.995 \\
\hline \multicolumn{5}{|c|}{ Dependent variable: AD-CT } \\
\hline Young adulthood & -0.002 & 0.970 & 0.008 & 0.894 \\
\hline Midlife & -0.017 & 0.779 & -0.001 & 0.987 \\
\hline Current & -0.117 & 0.088 & -0.033 & 0.586 \\
\hline
\end{tabular}

Adjusted for age, gender, education, apolipoprotein E $\varepsilon 4$ status, vascular risk score, use of sleep pill, Hamilton Depression Rating Score. AD-CT were natural log-transformed to achieve normal distribution

$A D$-CM Alzheimer's disease signature region cerebral glucose metabolism, $A D$ CT Alzheimer's disease signature region cortical thickness

${ }^{\text {a }}$ Positive beta value means that better sleep quality or decreased duration of sleep is associated with decreased glucose metabolism and/or cortical thickness, respectively
Several studies have provided clues regarding the plausible mechanisms that may underlie the influence of sleep disturbances on cerebral $A \beta$ accumulation. $A \beta$ levels in the brain interstitial fluid (ISF) are directly increased by elevations in synaptic activity [33], which decreases during sleep [34]. Therefore, disturbed sleep could increase amyloid production via enhancements of synaptic activity. In terms of $A \beta$ clearance, natural sleep increases the exchange in cerebrospinal fluid with ISF, which results in an elevated rate of $A \beta$ clearance [35].

Regarding neurodegeneration, we found that short sleep duration during midlife was associated with decreased glucose metabolism in the brain regions that are vulnerable to $\mathrm{AD}[27,28]$; this finding remained significant even after controlling for global $A \beta$ deposition. Evidence from experimental as well as clinical studies indicates that insufficient sleep, whether it is behavioral or disease-related, may stimulate appetite and cause disturbances in glucose metabolism [36]. Moreover, several studies have demonstrated that higher fasting serum glucose levels [37] or higher glycated hemoglobin levels [38] in non-diabetic CN older adults are associated with cerebral hypometabolism in AD signature brain regions. Furthermore, wakefulness promotes neurotoxic and oxidative stresses that are related to neurodegeneration, which increases the risk for $\mathrm{AD}$ [39]. Overall, the present results provide additional insights regarding the manner in which short sleep duration during the midlife period could contribute to AD-related cerebral hypometabolism via an amyloid-independent mechanism.

In contrast to the findings regarding cerebral $A \beta$ deposition and regional hypometabolism, there were no significant associations between sleep experiences during any of the lifetime periods and cortical thickness in the $\mathrm{AD}$ signature regions. Only a few studies have investigated the associations between brain structures and sleep in healthy individuals. These studies have found that a reduced volume in the orbitofrontal cortex (OFC) is linked with increased early morning awakenings [40] and daytime sleepiness [41]. Furthermore, increased atrophy in the superior frontal cortex [15] and insular region [9] is associated with poor sleep quality. In the 
present study, additional analyses were performed assessing the previously identified brain regions, including the OFC, superior frontal cortex, and insula, but no significant associations were observed (Additional file 1: Table S2). These discrepancies may be related to the differences in subject characteristics and analysis methods among studies. Although the present study included only elderly participants, previous studies $[9,15,40,41]$ included a wide range of ages with a younger average age. Because normal aging itself influences atrophy in the prefrontal cortex, including the OFC [42], our negative results can be interpreted to mean that the association between poor sleep and brain atrophy was relatively diminished due to the aging effects in elderly individuals. Another possibility for this discrepancy could be the use of different image analysis methods; we employed an ROI-based approach whereas previous studies $[9,15,40,41]$ have used voxelbased methods.

The strengths of the present study include the evaluation of sleep experiences in different lifetime periods, the use of multimodal neuroimaging, and the recruitment of healthy elderly people with fewer depressive symptoms and few sleep disorders. However, several limitations should also be mentioned. First, we included only subjective measurements of sleep experiences and did not employ objective sleep measures. However, there is a modest relationship between self-reported sleep experiences and more objective measures using polysomnography $[21,43]$. Apart from its relevance to the objectively measured sleep, self-reported sleep experiences are important because they are strongly associated with the risk for $\mathrm{AD}$ dementia [3] and $\mathrm{A} \beta$ burden [8-11], which was also observed in the present study. Furthermore, sleep questionnaires are advantageous in that they are feasible, easily used in clinical practice, and directly reflect one's perception of their own sleep, which may incorporate their actual sleep patterns as well as the emotional response to sleep [44, 45]. Second, although all efforts were made to overcome the limitations inherent to cross-sectional studies and the lifetime sleep experiences were separately assessed over three periods of life, all data on sleep experiences were obtained based on individual recall. The recall bias of sleep assessment increases with poor cognitive function [46] and severe depressive symptom [47]. To reduce this bias as much as possible, only $\mathrm{CN}$ elderly subjects without depressive disorders were included in this study. As a result, the overall age-related pattern of self-reported sleep in the present subjects (Table 1) matched well with the established age-related pattern of sleep based on objective measurement, i.e., sleep quality got poorer and sleep duration became shorter as the age of the participants increased [48]. If recall bias occurs randomly, then it is likely that the relationships between sleep and the biomarkers of $\mathrm{AD}$ were underestimated. Therefore, our positive findings were not likely to be observed by chance. Finally, it is possible that the bad sleep experiences were related to other unmeasured etiological factors, such as poor sleep hygiene, psychological factors, and/or other subclinical medical conditions, which in and of themselves may be linked to $\mathrm{AD}$ processes.

\section{Conclusions}

Our results suggest that bad sleep quality during midlife increases pathological $A \beta$ deposition in the brain and that short sleep duration during the same period accelerates AD-related regional hypometabolism. On the other hand, the present results do not support the hypothesis that $A \beta$ deposition or the neurodegenerative process influences sleep experiences in cognitively healthy old adults.

\section{Additional file}

Additional file 1: Table S1. Multiple linear regression analyses with neurodegeneration biomarkers as dependent variable after controlling for covariates including A $\beta$ burden, Table S2. Multiple linear regression analyses with cortical thickness of other than AD-signature regions as dependent variables and list of co-investigators (KBASE research group). (DOCX $35 \mathrm{~kb}$ )

\section{Abbreviations}

AD: Alzheimer's disease; AD-CM: Alzheimer's disease signature region cerebral glucose metabolism; AD-CT: Alzheimer's disease signature region cortical thickness; AD-ND: Alzheimer's disease signature neurodegeneration; APOE: Apolipoprotein E; APOE4: Apolipoprotein E ع4; Aß: Amyloid-beta; CERAD-K: Consortium to Establish a Registry for Alzheimer's Disease, Korean version; CN: Cognitively normal; FDG: $\left[{ }^{18} \mathrm{~F}\right]$ Fluorodeoxyglucose;

HDRS: Hamilton Depression Rating Scale; ISF: Interstitial fluid; KBASE: Korean Brain Aging Study for the Early Diagnosis and Prediction of AD; MRI: Magnetic resonance imaging; OFC: Orbitofrontal cortex; PET: Positron emission tomography; PiB: $\left[{ }^{11} \mathrm{C}\right]$ Pittsburgh compound B; PSQI: Pittsburgh Sleep Quality Index; ROIs: Region of interests; SUVR: Standardized uptake value ratio; VRS: Vascular risk score

\section{Acknowledgements}

We thank all study participants and their families for their contribution.

\section{Authors' contributions}

YMC was responsible for the acquisition, analysis, and interpretation of the data and drafting and critical revision of the manuscript for intellectual content. MSB, DY, JHL, and SYJ participated in the acquisition, analysis, and interpretation of the data. BKS, YKK, SAS, CS, and YJL participated in the analysis and interpretation of the data. DYL was responsible for the study concept and design; acquisition, analysis, and interpretation of the data; and drafting and critical revision of the manuscript for intellectual content. All authors read and approved the final manuscript.

\section{Funding}

This study was supported by a grant from the Ministry of Science, ICT, and Future Planning, Republic of Korea (Grant No: NRF-2014M3C7A1046042) and a grant of the Korea Health Technology R\&D Project through the Korea Health Industry Development Institute (KHIDI), funded by the Ministry of Health \& Welfare, Republic of Korea (Grant No: HI18C0630). The funding source had no role in the design of the study; collection, analysis, and interpretation of the data; and writing of the manuscript. 


\section{Availability of data and materials}

The datasets that were generated and analyzed during the present study are not publicly available due to ethical considerations and privacy restrictions. Data may be obtained from the corresponding author upon approval of the Institutional Review Board of the Seoul National University Hospital, Republic of Korea.

\section{Ethics approval and consent to participate}

The study protocol was approved by the Institutional Review Board of Seoul National University Hospital, and written informed consent was obtained from all participants.

\section{Consent for publication}

Not applicable

\section{Competing interests}

The authors declare that they have no competing interests.

\section{Author details}

'Department of Neuropsychiatry, Hallym University Dongtan Sacred Heart Hospital, Hwaseong, Republic of Korea. ${ }^{2}$ Institute of Human Behavioral Medicine, Medical Research Center, Seoul National University, Seoul, Republic of Korea. ${ }^{3}$ Department of Neuropsychiatry, Seoul National University Hospital, Seoul, Republic of Korea. ${ }^{4}$ Department of Neuropsychiatry, Chungnam National University Hospital, Daejeon, Republic of Korea. ${ }^{5}$ Department of Psychiatry, Sanggye Paik Hospital, Inje University College of Medicine, Seoul, Republic of Korea. ${ }^{6}$ Department of Nuclear Medicine, SMG-SNU Boramae Medical Center, Seoul, Republic of Korea. ${ }^{7}$ Department of Radiology, Seoul National University Hospital, Seoul, Republic of Korea. ${ }^{8}$ Center for Sleep and Chronobiology, Seoul National University Hospital, Seoul, Republic of Korea. ${ }^{9}$ Department of Psychiatry, Seoul National University College of Medicine, Seoul, Republic of Korea

Received: 16 May 2019 Accepted: 27 August 2019

Published online: 12 September 2019

\section{References}

1. Vitiello MV, Prinz PN, Williams DE, Frommlet MS, Ries RK. Sleep disturbances in patients with mild-stage Alzheimer's disease. J Gerontol. 1990;45(4): M131-8.

2. Lim AS, Kowgier M, Yu L, Buchman AS, Bennett DA. Sleep fragmentation and the risk of incident Alzheimer's disease and cognitive decline in older persons. Sleep. 2013:36(7):1027-32.

3. Hahn EA, Wang HX, Andel R, Fratiglioni L. A change in sleep pattern may predict Alzheimer disease. Am J Geriatr Psychiatry. 2014;22(11):1262-71.

4. Ju YE, Lucey BP, Holtzman DM. Sleep and Alzheimer disease pathology-a bidirectional relationship. Nat Rev Neurol. 2014;10(2):115-9.

5. Roh JH, Huang Y, Bero AW, Kasten T, Stewart FR, Bateman RJ, et al. Disruption of the sleep-wake cycle and diurnal fluctuation of beta-amyloid in mice with Alzheimer's disease pathology. Sci Transl Med. 2012;4(150): 150ra22.

6. Kang JE, Lim MM, Bateman RJ, Lee JJ, Smyth LP, Cirrito JR, et al. Amyloidbeta dynamics are regulated by orexin and the sleep-wake cycle. Science. 2009:326(5955):1005-7.

7. Slats D, Claassen JA, Verbeek MM, Overeem S. Reciprocal interactions between sleep, circadian rhythms and Alzheimer's disease: focus on the role of hypocretin and melatonin. Ageing Res Rev. 2013;12(1):188-200.

8. Spira AP, Gamaldo AA, An Y, Wu MN, Simonsick EM, Bilgel M, et al. Selfreported sleep and beta-amyloid deposition in community-dwelling older adults. JAMA Neurol. 2013;70(12):1537-43.

9. Branger P, Arenaza-Urquijo EM, Tomadesso C, Mezenge F, Andre C, de Flores $\mathrm{R}$, et al. Relationships between sleep quality and brain volume, metabolism, and amyloid deposition in late adulthood. Neurobiol Aging 2016;41:107-14.

10. Brown BM, Rainey-Smith SR, Villemagne VL, Weinborn M, Bucks RS, Sohrabi $H R$, et al. The relationship between sleep quality and brain amyloid burden. Sleep. 2016:39(5):1063-8.

11. Sprecher KE, Bendlin BB, Racine AM, Okonkwo OC, Christian BT, Koscik RL, et al. Amyloid burden is associated with self-reported sleep in nondemented late middle-aged adults. Neurobiol Aging. 2015;36(9):2568-76.
12. Ju YE, McLeland JS, Toedebusch CD, Xiong C, Fagan AM, Duntley SP, et al. Sleep quality and preclinical Alzheimer disease. JAMA Neurol. 2013;70(5):587-93.

13. Ooms S, Overeem S, Besse K, Rikkert MO, Verbeek M, Claassen JA. Effect of 1 night of total sleep deprivation on cerebrospinal fluid beta-amyloid 42 in healthy middle-aged men: a randomized clinical trial. JAMA Neurol. 2014; 71(8):971-7.

14. Ju YS, Ooms SJ, Sutphen C, Macauley SL, Zangrilli MA, Jerome G, et al. Slow wave sleep disruption increases cerebrospinal fluid amyloid-beta levels. Brain. 2017;140(8):2104-11.

15. Sexton CE, Storsve AB, Walhovd KB, Johansen-Berg H, Fjell AM. Poor sleep quality is associated with increased cortical atrophy in community-dwelling adults. Neurology. 2014;83(11):967-73.

16. Byun MS, Yi D, Lee JH, Choe YM, Sohn BK, Lee JY, et al. Korean brain aging study for the early diagnosis and prediction of Alzheimer's disease: methodology and baseline sample characteristics. Psychiatry Investig. 2017; 14(6):851-63.

17. Lee JH, Lee KU, Lee DY, Kim KW, Jhoo JH, Kim JH, et al. Development of the Korean version of the Consortium to Establish a Registry for Alzheimer's Disease Assessment Packet (CERAD-K): clinical and neuropsychological assessment batteries. J Gerontol Ser B Psychol Sci Soc Sci. 2002;57(1):P47-53.

18. Lee DY, Lee KU, Lee JH, Kim KW, Jhoo JH, Kim SY, et al. A normative study of the CERAD neuropsychological assessment battery in the Korean elderly. J Int Neuropsychol Soc. 2004;10(1):72-81.

19. DeCarli C, Mungas D, Harvey D, Reed B, Weiner M, Chui H, et al. Memory impairment, but not cerebrovascular disease, predicts progression of $\mathrm{MCl}$ to dementia. Neurology. 2004;63(2):220-7.

20. Hamilton M. Development of a rating scale for primary depressive illness. $\mathrm{Br}$ J Soc Clin Psychol. 1967;6(4):278-96.

21. Buysse DJ, Reynolds CF 3rd, Monk TH, Berman SR, Kupfer DJ. The Pittsburgh Sleep Quality Index: a new instrument for psychiatric practice and research. Psychiatry Res. 1989;28(2):193-213.

22. Park JC, Han SH, Yi D, Byun MS, Lee JH, Jang S, et al. Plasma tau/amyloidbeta1-42 ratio predicts brain tau deposition and neurodegeneration in Alzheimer's disease. Brain. 2019;142(3):771-86.

23. Tzourio-Mazoyer N, Landeau B, Papathanassiou D, Crivello F, Etard O, Delcroix $\mathrm{N}$, et al. Automated anatomical labeling of activations in SPM using a macroscopic anatomical parcellation of the MNI MRI single-subject brain. Neurolmage. 2002;15(1):273-89.

24. Reiman EM, Chen K, Liu X, Bandy D, Yu M, Lee W, et al. Fibrillar amyloidbeta burden in cognitively normal people at 3 levels of genetic risk for Alzheimer's disease. Proc Natl Acad Sci U S A. 2009;106(16):6820-5.

25. Choe YM, Sohn BK, Choi HJ, Byun MS, Seo EH, Han JY, et al. Association of homocysteine with hippocampal volume independent of cerebral amyloid and vascular burden. Neurobiol Aging. 2014;35(7):1519-25.

26. Villeneuve S, Rabinovici GD, Cohn-Sheehy BI, Madison C, Ayakta N, Ghosh PM, et al. Existing Pittsburgh compound-B positron emission tomography thresholds are too high: statistical and pathological evaluation. Brain. 2015; 138(Pt 7):2020-33.

27. Jack CR Jr, Wiste HJ, Weigand SD, Rocca WA, Knopman DS, Mielke MM, et al. Age-specific population frequencies of cerebral beta-amyloidosis and neurodegeneration among people with normal cognitive function aged 5089 years: a cross-sectional study. Lancet Neurol. 2014;13(10):997-1005.

28. Jack CR Jr, Wiste HJ, Weigand SD, Knopman DS, Mielke MM, Vemuri $P$, et al. Different definitions of neurodegeneration produce similar amyloid/ neurodegeneration biomarker group findings. Brain. 2015:138(Pt 12):3747-59.

29. Jagust WJ, Landau SM, Shaw LM, Trojanowski JQ, Koeppe RA, Reiman EM, et al. Relationships between biomarkers in aging and dementia. Neurology. 2009;73(15):1193-9.

30. Wenham PR, Price WH, Blandell G. Apolipoprotein E genotyping by onestage PCR. Lancet. 1991;337(8750):1158-9.

31. Spira AP, Yager C, Brandt J, Smith GS, Zhou Y, Mathur A, et al. Objectively measured sleep and beta-amyloid burden in older adults: a pilot study. SAGE Open Med. 2014;2. https://doi.org/10.1177/2050312114546520.

32. Hirshkowitz M, Whiton K, Albert SM, Alessi C, Bruni O, DonCarlos L, et al. National Sleep Foundation's sleep time duration recommendations: methodology and results summary. Sleep Health. 2015:1(1):40-3.

33. Cirrito JR, Yamada KA, Finn MB, Sloviter RS, Bales KR, May PC, et al. Synaptic activity regulates interstitial fluid amyloid-beta levels in vivo. Neuron. 2005; 48(6):913-22.

34. Vyazovskiy W, Olcese U, Lazimy YM, Faraguna U, Esser SK, Williams JC, et al. Cortical firing and sleep homeostasis. Neuron. 2009;63(6):865-78. 
35. Xie L, Kang H, Xu Q, Chen MJ, Liao Y, Thiyagarajan M, et al. Sleep drives metabolite clearance from the adult brain. Science. 2013;342(6156):373-7.

36. Hanlon EC, Van Cauter E. Quantification of sleep behavior and of its impact on the cross-talk between the brain and peripheral metabolism. Proc Natl Acad Sci U S A. 2011;108(Suppl 3):15609-16.

37. Burns CM, Chen K, Kaszniak AW, Lee W, Alexander GE, Bandy D, et al. Higher serum glucose levels are associated with cerebral hypometabolism in Alzheimer regions. Neurology. 2013;80(17):1557-64.

38. Byun MS, Kim HJ, Yi D, Choi HJ, Baek H, Lee JH, et al. Differential effects of blood insulin and HbA1c on cerebral amyloid burden and neurodegeneration in nondiabetic cognitively normal older adults. Neurobiol Aging. 2017;59:15-21.

39. Villafuerte G, Miguel-Puga A, Rodriquez EM, Machado S, Manjarrez E, AriasCarrion O. Sleep deprivation and oxidative stress in animal models: a systematic review. Oxidative Med Cell Longev. 2015:2015:234952.

40. Stoffers D, Moens S, Benjamins J, van Tol MJ, Penninx BW, Veltman DJ, et al. Orbitofrontal gray matter relates to early morning awakening: a neural correlate of insomnia complaints? Front Neurol. 2012;3:105.

41. Killgore WD, Schwab ZJ, Kipman M, DelDonno SR, Weber M. Voxel-based morphometric gray matter correlates of daytime sleepiness. Neurosci Lett. 2012;518(1):10-3.

42. Fjell AM, McEvoy L, Holland D, Dale AM, Walhovd KB, Alzheimer's Disease Neuroimaging I. Brain changes in older adults at very low risk for Alzheimer's disease. J Neurosci. 2013;33(19):8237-42.

43. Coates TJ, Killen JD, George J, Marchini E, Silverman S, Thoresen C. Estimating sleep parameters: a multitrait-multimethod analysis. J Consult Clin Psychol. 1982;50(3):345-52.

44. Vitiello MV, Larsen LH, Moe KE. Age-related sleep change: gender and estrogen effects on the subjective-objective sleep quality relationships of healthy, noncomplaining older men and women. J Psychosomatic Res. 2004;56(5):503-10.

45. Regestein QR, Friebely J, Shifren JL, Scharf MB, Wiita B, Carver J, et al. Selfreported sleep in postmenopausal women. Menopause. 2004;11(2):198-207.

46. Van Den Berg JF, Van Rooij FJ, Vos H, Tulen JH, Hofman A, Miedema HM, et al. Disagreement between subjective and actigraphic measures of sleep duration in a population-based study of elderly persons. J Sleep Res. 2008; 17(3):295-302.

47. Baillet M, Cosin C, Schweitzer P, Peres K, Catheline G, Swendsen J, et al. Mood influences the concordance of subjective and objective measures of sleep duration in older adults. Front Aging Neurosci. 2016;8:181.

48. Cooke JR, Ancoli-Israel S. Normal and abnormal sleep in the elderly. Handb Clin Neurol. 2011:98:653-65.

\section{Publisher's Note}

Springer Nature remains neutral with regard to jurisdictional claims in published maps and institutional affiliations.

Ready to submit your research? Choose BMC and benefit from:

- fast, convenient online submission

- thorough peer review by experienced researchers in your field

- rapid publication on acceptance

- support for research data, including large and complex data types

- gold Open Access which fosters wider collaboration and increased citations

- maximum visibility for your research: over $100 \mathrm{M}$ website views per year

At $\mathrm{BMC}$, research is always in progress.

Learn more biomedcentral.com/submissions 